\title{
RELAXATION OSCILLATIONS OF A VAN DER POL EQUATION WITH LARGE CRITICAL FORCING TERM*
}

\author{
BY \\ J. GRASMAN \\ Mathematical Centre, Amsterdam
}

\begin{abstract}
A van der Pol equation with sinusoidal forcing term is analyzed with singular perturbation methods for large values of the parameter. Asymptotic approximations of (sub)harmonic solutions with period $T=2 \pi(2 n-1), n=1,2, \ldots$ are constructed under certain restricting conditions for the amplitude of the forcing term. These conditions are such that always two solutions with period $T=2 \pi(2 n \pm 1)$ coexist.
\end{abstract}

1. Introduction. In this paper we consider a van der Pol equation for large parameter values with a periodic forcing term of a same order of magnitude:

$$
\frac{d^{2} x}{d t^{2}}+v\left(x^{2}-1\right) \frac{d x}{d t}+x=b(v) \cos t, \quad v \gg 1,
$$

with $b(v)=O(v)$. This equation was investigated with analytical-topological methods by Littlewood [8], who proved the existence of (sub)harmonic solutions of period

$$
T=2 \pi(2 n-1), \quad n=1,2, \ldots
$$

Littlewood stated that for $b=\alpha v, \alpha>2 / 3$ only globally asymptotically stable solutions of the period $2 \pi$ are found (see also [9]). The proof of this statement has been given by Lloyd [10]. For decreasing $\alpha$ there also occur solutions of period $6 \pi$. As $\alpha$ decreases further the $2 \pi$-periodic solution disappears, and $\alpha$ passes alternately intervals where one subharmonic solution of period $T=2 \pi(2 n-1)$ exists and intervals where two subharmonic solutions of period $T=2 \pi(2 n \pm 1)$ coexist $(n=1,2, \ldots)$. There are also intervals of more complicated behavior.

In Fig. 1 we give the overlapping domains $\Omega_{n}$ in the $(b / v, v)$-plane where a solution of period (1.2) with $n \leq 4$ is found. The figure is based on numerical results obtained by Flaherty and Hoppensteadt [2] for $1 / v>.01$. We see that for $v \rightarrow \infty$ these domains tend to a common boundary point $(b / v, v)=(2 / 3, \infty)$. In this paper we will analyze the local structure of the domains $\Omega_{n}$ near $(b / v, v)=(2 / 3, \infty)$. For that purpose we write

$$
b=\alpha v+\beta \text {. }
$$

Using singular perturbation techniques, we will construct asymptotic approximations of

\footnotetext{
* Received March 13, 1979; revised version received May 2, 1979. The author is grateful to E. J. M. Veling for reading the manuscript and for some valuable remarks.
} 


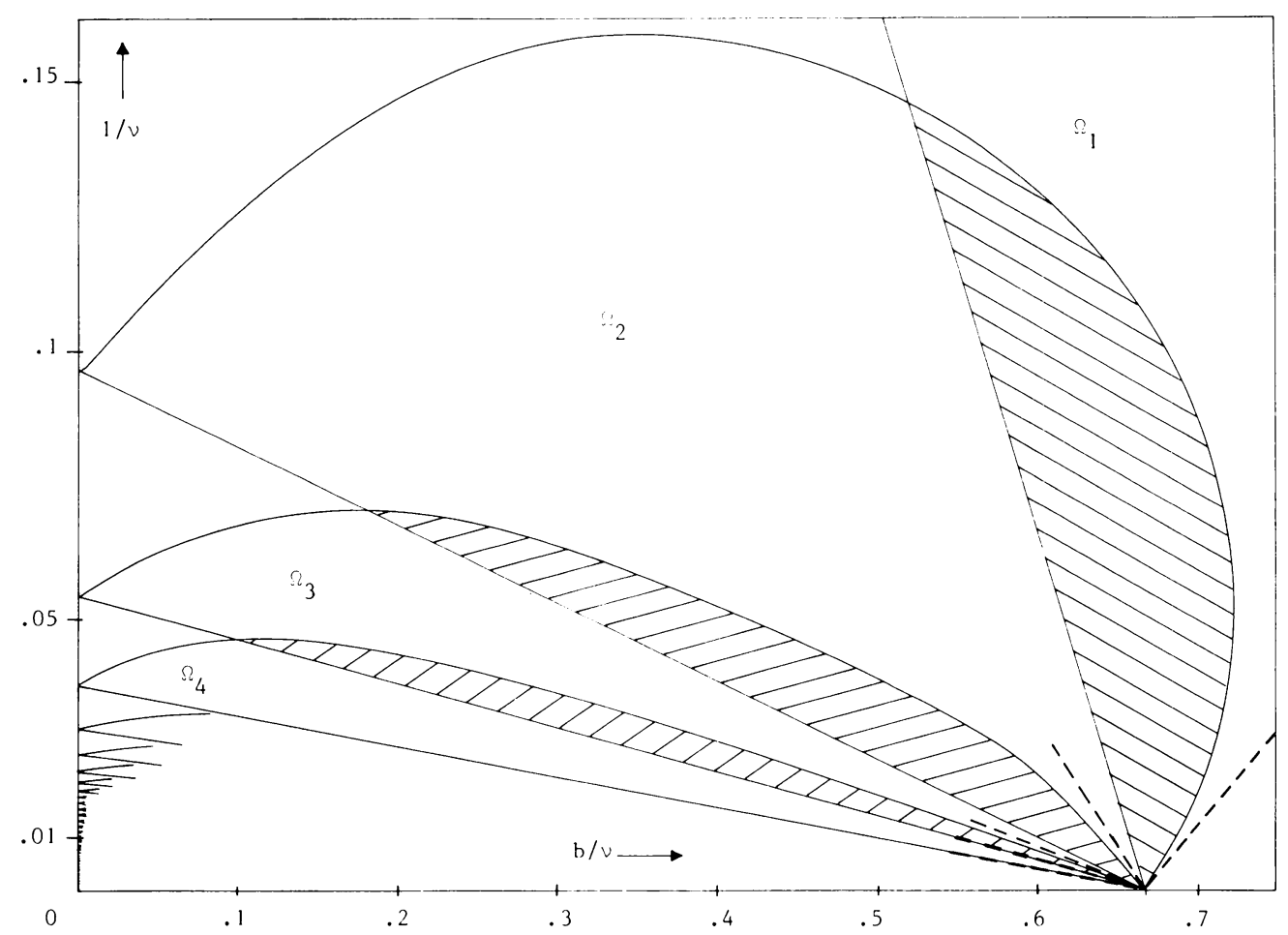

FIG. 1. The domains $\Omega_{n}$ with a periodic solution $T=2 \pi(2 n-1)$.

(sub)harmonic solutions of (1.1), (1.3) with $\alpha=2 / 3$. The periods of these solutions satisfy (1.2) with $n$ independent of $v$. In the process of construction of the approximation we will have to impose conditions upon $\beta$ of the type

$$
\underline{\beta}_{n}<\beta<\bar{\beta}_{n}
$$

to approximate symmetric solutions of period $T=2 \pi(2 n-1)$. It turns out that

$$
\underline{\beta}_{n}<\bar{\beta}_{n+1}=\underline{\beta}_{n-1}<\bar{\beta}_{n},
$$

so that near $(b / v, v)=(2 / 3, \infty)$ the domains $\Omega_{n}$ overlap as sketched in Fig. 2 .

This overlapping of intervals differs slightly from analytical results $[5,6,8]$, as we only find intervals with two subharmonic solutions of period $T=2 \pi(2 n \pm 1)$. In $[3,4]$ the case $\alpha=0$ was also analyzed with asymptotic techniques. There the subharmonics had a period $T=2 \pi n$ with $n=\mathrm{O}(v)$. The choice $\alpha=0$ or $\alpha=2 / 3$ leads to solutions with completely different asymptotic behavior, and this makes it necessary to consider them as separate problems. In [3] we met an unusual structure of two-variable expansions matched with boundary layer solutions. We will see here that the case $\alpha=2 / 3$ also exhibits an exceptional structure. The global behavior of the solution depends strongly on local conditions: each time the solutions pass a neighborhood of the lines $x= \pm 1$ some quantity is increased by a given value. When this quantity, being an integration constant in the local asymptotic solution, reaches a threshold value the solution enters a phase of rapid change characteristic of a relaxation oscillation. This part of the solution is approximated by a boundary layer type of solution. For the regions sketched in Fig. 3 


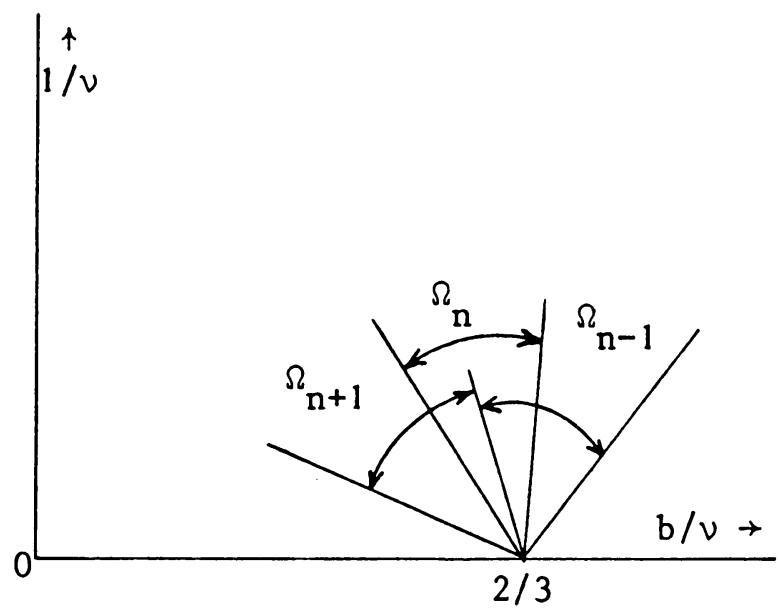

Fig. 2. Local structure of the domains $\Omega_{n}$ near $(b / v, v)=(2 / 3, \infty)$ derived from the formal asymptotic analysis. Exact values are given in Fig. 1 (dotted lines) for $n \leq 4$.

separate local approximations have been constructed from the differential equation. Integration constants in these local asymptotic solutions are determined by matching pairs of local solutions of adjacent regions.

Thus, in this paper we investigate the equation

$$
\frac{d^{2} x}{d t^{2}}+v\left(x^{2}-1\right) \frac{d x}{d t}+x=\left(\frac{2}{3} v+\beta\right) \cos t .
$$

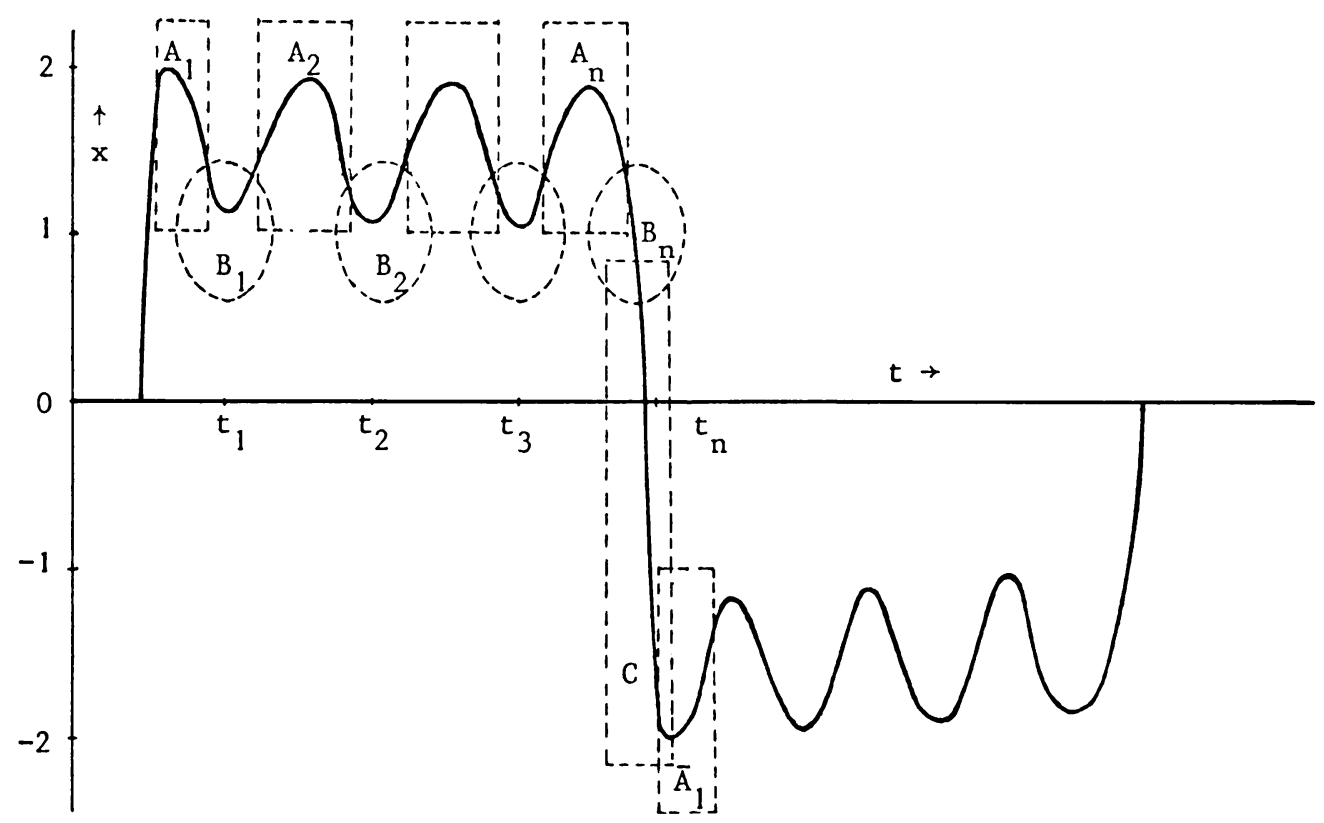

FIG. 3. Characteristic regions for a periodic solution of (1.6). 
It is expected that the study of this problem with a critical forcing term may bring us into a position to deal successfully with the more complicated problem of $0<\alpha<2 / 3$. It is anticipated that periodic solutions of this problem have a behavior in which elements of both the case $\alpha=0$ and $\alpha=2 / 3$ are present. It is remarked that the limit cases $\alpha=0$ and $\alpha=2 / 3$ are not covered by the recent analytical studies $([5,6,10])$, where, respectively, $0<\alpha<2 / 3$ and $\alpha>2 / 3$.

2. Asymptotic solutions for the regions $A_{m}$. It is supposed that in the regions $A_{m}$ where $1<x<2$ the solution can be expanded as

$$
x(t ; \varepsilon)=x_{m 0}(t)+v^{-1} x_{m 1}(t)+\cdots .
$$

Substituting (2.1) into Eq. (1.6) and equating the terms of order $O(v)$ and $O(1)$, we obtain

$$
\begin{gathered}
\left(x_{m 0}^{2}-1\right) \frac{d x_{m 0}}{d t}=\frac{2}{3} \cos t, \\
\left(x_{m 0}^{2}-1\right) \frac{d x_{m 1}}{d t}+2 x_{m 0} x_{m 1} \frac{d x_{m 0}}{d t}=-\frac{d x_{m 0}^{2}}{d t^{2}}-x_{m 0}+\beta \cos t .
\end{gathered}
$$

Integration of Eq. (2.2) gives

$$
\frac{1}{3} x_{m 0}^{3}-x_{m 0}=\frac{2}{3} \sin t+C_{0}^{(m)} .
$$

Since in the regions $A_{m}$ the value of the left-hand side of this equation varies from $-2 / 3$ to $2 / 3$, we have to take $C_{0}^{(m)}=0$. For this value of $C_{0}^{(m)}$ the solutions of (2.4) read

$$
x_{m 0}(t)=2 \cos \left\{\frac{1}{3}(\arccos (\sin t)+2 \pi j)\right\}, \quad j=0,1,2 .
$$

As $x_{m 0}$ has to be within the interval $(1,2)$, we select the branch with $j=0$. Integrating (2.3), while making use of (2.2), we obtain

$$
\begin{aligned}
\left(x_{m 0}^{2}-1\right) x_{m 1} & =-\frac{2 \cos t}{3\left(x_{m 0}^{2}-1\right)}-\int_{t_{m-1}}^{t} x_{m 0}(\tau) d \tau+\beta \sin t+C_{1}^{(m)}, \\
t_{m} & =2 \pi m-\pi / 2 .
\end{aligned}
$$

When $t$ approaches $t_{m}$ from below, $x_{m 0}$ and $x_{m 1}$ behave as

$$
x_{m 0} \approx 1-\left(t-t_{m}\right) / \sqrt{ } 3, \quad x_{m 1} \approx K_{m} /\left(t-t_{m}\right),
$$

where

$$
K_{m}=-\frac{1}{2}+\frac{1}{2} \sqrt{ } 3\left(-C_{1}^{(m)}+I\right), \quad I=\int_{t_{m-1}}^{t_{m}} x_{m 0}(t) d t=6 \sqrt{ } 3 .
$$

Thus, for $t \uparrow t_{m}$ the asymptotic solution (2.1) loses its validity.

3. Asymptotic solution for the regions $B_{m}$. We analyze the local behavior of the solution near $(x, t)=\left(1, t_{m}\right), m=1,2, \ldots$ by introducing a stretching transformation in both the dependent and independent variable:

$$
x=1+V_{m}(\xi) v^{-i}, \quad t=t_{m}+\xi v^{-x} .
$$


Substitution into the differential equation yields

$$
\begin{aligned}
v^{-\gamma+2 \alpha} \frac{d^{2} V_{m}}{d \xi^{2}}+v^{1-2 \gamma+\alpha}\left(2 V_{m}+v^{-\gamma} V_{m}^{2}\right) \frac{d V_{m}}{d \xi}+1+ & V_{m} v^{-\gamma} \\
& =\left(\frac{2}{3} v+\beta\right)\left(\xi v^{-\alpha}-\frac{\xi^{3} v^{-3 \alpha}}{3 !}+\cdots\right) .
\end{aligned}
$$

We see that for $\alpha=\gamma=1 / 2$ the second derivative becomes of the same order of magnitude in $v$ as the leading terms constituting Eq. (2.2). Multiplying the equation by $v^{-1 / 2}$ and letting $v$ tend to infinity, we obtain the limit equation

$$
\frac{d^{2} V_{m 0}}{d \xi^{2}}+2 V_{m 0} \frac{d V_{m 0}}{d \xi}=\frac{2}{3} \xi
$$

The function $V_{m 0}(\xi)$ expresses the local limit behavior of the solution for $v \rightarrow \infty$. In order to match the solution of region $A_{m}$ it must satisfy

$$
V_{m 0}(\xi) \approx \frac{\xi}{\sqrt{3}}+\frac{K_{m}}{\xi}
$$

for $\xi \rightarrow \infty$ (see (2.8)). Such a function indeed exists and has the form

$$
V_{m 0}(\xi)=a \frac{D_{K_{m}}^{\prime}(-a \xi)}{D_{K_{m}}(-a \xi)}, \quad a=\sqrt[4]{4 / 3}
$$

where $D_{\mu}(z)$ is the so-called parabolic cylinder function of order $\mu$ (see Whittaker and Watson [11, p. 347]). For $z \rightarrow \infty$ we have that

$$
\left.D_{\mu}(z)=\exp \left(-\frac{1}{4} z^{2}\right) z^{\mu} \mid 1-\frac{\mu(\mu-1)}{2 z^{2}}+\cdots\right\},
$$

while for $z \rightarrow-\infty$

$$
\begin{aligned}
D_{\mu}(z)= & \exp \left(-\frac{1}{4} z^{2}\right) z^{\mu}\left\{1-\frac{\mu(\mu-1)}{2 z^{2}}+\cdots\right\} \\
& \left.-\frac{\sqrt{2 \pi}}{\Gamma(-\mu)} \exp \left(\frac{1}{4} z^{2}\right) z^{-\mu-1} \mid 1+\frac{(\mu+1)(\mu+2)}{2 z^{2}}+\cdots\right\} .
\end{aligned}
$$

Assuming that $K_{m} \leq 0$, the function $V_{m 0}(\xi)$ will be regular for finite $\xi$, while for $\xi \rightarrow \infty$

$$
V_{m 0}(\xi) \approx \frac{\xi}{\sqrt{ } 3}-\frac{K_{m}+1}{\xi} .
$$

On the other hand, at region $A_{m+1}$ the solution is approximated by

$$
x(t)=1+\frac{\left(t-t_{m}\right)}{\sqrt{ } 3}+\frac{-\frac{1}{2}+\frac{1}{2} \sqrt{ } 3\left(C_{1}^{(m+1)}-\beta\right)}{\left(t-t_{m}\right)}+O\left(\left(t-t_{m}\right)^{-3}\right)
$$

as $t \downarrow t_{m}$. Consequently, (3.6) matches the local solution for region $A_{m+1}$ if

$$
K_{m}=-\frac{1}{2}+\frac{1}{2} \sqrt{ } 3\left(\beta-C_{1}^{(m+1)}\right)
$$


or, using $(2.9 \mathrm{a})$,

$$
C_{1}^{(m+1)}=C_{1}^{(m)}-I
$$

with $I=6 \sqrt{ } 3($ see $(2.9 \mathrm{~b}))$. Obviously, we will arrive in the situation that for some $m$, say $m=n$,

$$
K_{n-1} \leq 0<K_{n} \leq \frac{1}{2} \sqrt{ } 3 I
$$

(if $n=1$, inequality (3.8) reads $K_{1}>0$ ). The parabolic cylinder function $D_{\mu}(z)$ with $\mu>0$ vanishes for certain value(s) of the argument $z$. Let $z_{0}$ be the largest zero. For $\xi \uparrow \xi_{0}$ with $z_{0}=a \xi_{0}$ we have

$$
V_{m 0}(\xi) \approx\left(\xi-\xi_{0}\right)^{-1}+\frac{1}{3} a^{2}\left(\frac{1}{4} a^{2} \xi_{0}^{2}-K_{n}-\frac{1}{2}\right)\left(\xi-\xi_{0}\right),
$$

so $V_{n 0} \rightarrow-\infty$ and the local solution at region $B_{n}$ becomes singular at $\xi=\xi_{0}$.

4. Asymptotic solution for region $C$. At this point the solution enters the boundary layer region $C$ with local coordinate

$$
\eta=\left(t-t_{n}-\xi_{0} v^{-1 / 2}\right) v .
$$

We assume that the solution can be expanded as

$$
x=W_{0}(\eta)+v^{-1} W_{1}(\eta)+v^{-3 / 2} W_{2}(\eta)+\cdots .
$$

Substituting (4.1) and (4.2) into (1.6) and equating the terms of order $O\left(v^{2}\right)$ and $O(v)$ we obtain, respectively,

$$
\begin{aligned}
d^{2} W_{0} / d \eta^{2}+\left(W_{0}^{2}-1\right) d W_{0} / d \eta & =0, \\
d^{2} W_{1} / d \eta^{2}+\left(W_{0}^{2}-1\right) d W_{1} / d \eta+2 W_{0} W_{1}\left(d W_{0} / d \eta\right) & =0 .
\end{aligned}
$$

The solution of the first equation matches the local solution for region $B_{n}$ if

$$
W_{0}(\eta) \approx 1+1 / \eta
$$

as $\eta \rightarrow-\infty$ (see (3.9)). This condition is satisfied by the class of solutions

$$
\frac{1}{1-W_{0}}+\frac{1}{3} \log \frac{W_{0}+2}{1-W_{0}}=-\eta+H_{0},
$$

where the integration constant $H_{0}$ is found from matching with higher-order terms of the asymptotic solution for region $B_{n}$. It turns out that

$$
H_{0}=\frac{1}{6} \log v+\frac{1}{3} \log 3 .
$$

From (3.9) we also deduce that $W_{1}$ should behave as

$$
W_{1}(\eta) \approx \frac{1}{3} a^{2}\left(\frac{1}{4} a^{2} \xi_{0}^{2}-K_{n}-\frac{1}{2}\right) \eta
$$

for $\eta \rightarrow-\infty$, so that the integrated equation (4.4) will have the form

$$
\frac{d W_{1}}{d \eta}+\left(W_{0}^{2}-1\right) W_{1}=a^{2}\left(\frac{1}{4} a^{2} \xi_{0}^{2}-K_{n}-\frac{1}{2}\right) .
$$


On the other hand, for $\eta \gg 1 / 6 \log v$ we have

$$
\begin{aligned}
& W_{0}(\eta)=-2+O\left(v^{1 / 2} e^{-3 \eta}\right), \\
& W_{1}(\eta)=\frac{1}{3} a^{2}\left(\frac{1}{4} a^{2} \xi_{0}^{2}-K_{n}-\frac{1}{2}\right)+O\left(e^{-3 \eta}\right) .
\end{aligned}
$$

The boundary-layer solution matches the solution for region $\bar{A}_{1}$ if

$$
\begin{aligned}
\bar{x}_{10}\left(t_{n}+\xi_{0} v^{-1 / 2}\right)+v^{-1} \bar{x}_{11}\left(t_{n}+\xi_{0} v^{-1 / 2}\right) & \\
& =-2+\frac{1}{3} a^{2}\left(\frac{1}{4} a^{2} \xi_{0}^{2}-K_{n}-\frac{1}{2}\right) v^{-1}+o\left(v^{-1}\right),
\end{aligned}
$$

where $\bar{x}_{1 i}(t)$ are the coefficients of an expansion for region $\bar{A}_{1}$ of the form (2.1).

5. Periodicity conditions. Let us assume that the periodic solutions we are looking for are symmetric in the sense that $x(t)=-x\left(t-\frac{1}{2} T\right)$. Then we have completed the local approximations. Transposing (4.11) to the complementary phase $t=t_{1}-\pi+\xi_{0} v^{-1 / 2}$, in region $A_{1}$ we have

$$
\begin{aligned}
x_{10}\left(t_{1}-\pi+\xi_{0} v^{-1 / 2}\right)+v^{-1} x_{11}\left(t_{1}-\pi\right. & \left.+\xi_{0} v^{-1 / 2}\right) \\
& =2-\frac{1}{3} a^{2}\left(\frac{1}{4} a^{2} \xi_{0}^{2}-K_{n}-\frac{1}{2}\right) v^{-1}+o\left(v^{-1}\right)
\end{aligned}
$$

or

$$
K_{n}=-\frac{1}{2}+\frac{1}{2} \sqrt{ } 3\left(\beta+C_{1}^{(1)}-\frac{1}{2} I\right) .
$$

Using (2.9a), (3.7) and (5.2), we find

$$
\beta=\frac{1}{\sqrt{ } 3}\left(2 K_{n}+1\right)-\frac{1}{2}\left(n-\frac{1}{2}\right) I .
$$

From (3.8) we know that $K_{n}$ ranges from 0 to 9 , so $\beta$ has to satisfy

$$
3 \sqrt{ } 3\left(\frac{11}{18}-n\right)<\beta<3 \sqrt{ } 3\left(\frac{47}{18}-n\right), \quad n=2,3, \ldots
$$

Solutions of period $2 \pi$ are found for $\beta>-7 / 6 \sqrt{ } 3$.

6. Some remarks. Our asymptotic results hold for values of $\beta$ that are independent of $v$ and satisfy the inequality (5.4). Because of this the domains $\Omega_{n-1}$ and $\Omega_{n+1}$ are separated near $(b / v, v)=(2 / 3, \infty)$ by a sectorial domain $\Gamma_{n}$ of thickness $o(1)$ as $v \rightarrow \infty$. A qualitative analysis of (1.1), (1.3) with $0<\alpha<2 / 3$ by Levi [6] reveals the existence of infinitely many different periodic solutions for uncountably many values of $\beta$ near $\beta_{n}$ and $\bar{\beta}_{n}$. It is expected that at $\alpha=2 / 3$ a similar phenomenon occurs for values of $b$ and $v$ that are restricted to the domains $\Gamma_{n \pm 1}$. Furthermore, for $0<\alpha<2 / 3$ there are intervals $\bar{\beta}_{n+1}<\beta<\beta_{n-1}$, where only one solution with period $T=2 \pi(2 n-1)$ is found. Clearly, for $\alpha \rightarrow 2 / 3$ these intervals disappear and become part of the small transition intervals described above.

For the case $\alpha=0$ nonsymmetric solutions with period $T=4 \pi n$ have been constructed in [4], and it is also indicated there how the method can be extended to solutions with a rational rotation number. Trying to derive similar results for $\alpha=2 / 3$, we find that the symmetry condition of Sec. 5 is necessary. Thus, nonsymmetric solutions are only expected at the boundaries of the $\beta$-intervals. An asymptotic analysis of this problem requires elaborate calculations of higher-order approximations. 
Finally, it is remarked that the asymptotic solution of the case $0<\alpha<2 / 3$ should match the present results for $\alpha \rightarrow 2 / 3$ and the outcome of earlier work [3] for $\alpha \rightarrow 0$. This problem has not been solved yet. It is expected that it will give rise to some serious difficulties in the construction of the local asymptotic solution of a region of length $O(v)$, where the solution has a two time-scale behavior and satisfies asymptotically an equation of the type (2.4) with a slowly varying integration constant.

\section{REFERENCES}

[1] H. Bavinck and J. Grasman, The method of matched asymptotic expansions for the periodic solution of the Van der Pol equation, Int. J. Nonlin. Mech. 9, 421-434 (1974)

[2] J. E. Flaherty and F. C. Hoppensteadt, Frequency entrainment of a forced Van der Pol oscillator, Studies Appl. Math. 18, 5-15 (1978)

[3] J. Grasman, E. J. M. Veling and G. M. Willems, Relaxation oscillations governed by a Van der Pol equation with periodic forcing term, SIAM J. Appl. Math. 31, 667-676 (1976)

[4] J. Grasman, M. J. W. Jansen and E. J. M. Veling, Asymptotic methods for relaxation oscillations, in Proceedings of the third Scheveningen conference on differential equations, W. Eckhaus and E. M. Jager (eds.), North Holland Math. Studies 31, 93-111 (1978)

[5] J. Guckenheimer, Symbolic dynamics and relaxation oscillations, preprint

[6] M. Levi, Qualitative analysis of the periodically forced relaxation oscillations, Ph.D. Thesis, New York University, New York (1978)

[7] N. Levinson, A second-order differential equation with singular solutions, Ann. Math. 50, 127-152 (1949)

[8] J. E. Littlewood, On nonlinear differential equations of the second order, III, Acta Math. 97, 267-308 (1957)

[9] J. E. Littlewood, On Van der Pol's equation with large $k$, in Nonlinear problems, R. E. Langer (ed.), Univ. of Wisconsin Press, Madison, 161-165 (1963)

[10] N. G. Lloyd, On the non-autonomous Van der Pol equation with large parameter, Proc. Camb. Phil. Soc. 72 , 213-227 (1972)

[11] E. T. Whittaker and G. N. Watson, A course of modern analysis, Cambridge, Cambridge University Press (1935) 